\title{
Conformastatic disk-haloes in Einstein-Maxwell gravity
}

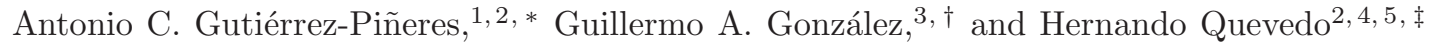 \\ ${ }^{1}$ Facultad de Ciencias Básicas, Universidad Tecnológica de Bolívar, Cartagena, Colombia \\ ${ }^{2}$ Instituto de Ciencias Nucleares, Universidad Nacional Autónoma de México, AP 70543, México, DF 04510, México \\ ${ }^{3}$ Escuela de Fúsica, Universidad Industrial de Santander, A. A. 678, Bucaramanga, Colombia \\ ${ }^{4}$ Instituto de Cosmologia, Relatividade e Astrofisica ICRA - CBPF \\ Rua Dr. Xavier Sigaud, 150, CEP 22290-180, Rio de Janeiro, Brazil \\ ${ }^{5}$ Dipartimento di Fisica and Icra, Università di Roma La Sapienza, I-00185 Roma, Italy
}

We present a relativistic model describing a thin disk surrounded by a halo in presence of an electromagnetic field. The model is obtained by solving the Einstein-Maxwell equations on a particular conformastatic spacetime background and by using the distributional approach for the energymomentum tensor. A class of solutions is obtained in which the gravitational and electromagnetic potentials are completely determined by a harmonic function only. A particular solution is given that is asymptotically flat and singularity-free, and satisfies all the energy conditions.

PACS numbers: 04.20.-q, 04.20.Jb, 04.40.-b, 04.40.Nr

\footnotetext{
* e-mail acgutierrez@correo.nucleares.unam.mx

† e-mail guillego@uis.edu.co

$\ddagger$ e-mail quevedo@nucleares.unam.mx
} 


\section{INTRODUCTION}

A large number of galaxies and other astrophysical systems have extended mass distributions surrounded by a material halo. For practical reasons one can assume that many of these systems preserve axial symmetry and, therefore, they can be modeled in terms of relativistic thin disks with exterior halos, in particular when the gravity field is strong enough. Disks may also be used to model accretion disks, galaxies in thermodynamic equilibrium and the superposition of a black hole and a galaxy. Disk sources for stationary axially symmetric spacetimes with magnetic fields are also of astrophysical importance mainly in the study of neutron stars, white dwarfs and galaxy formation. To describe the gravitational and electromagnetic fields of such configurations, we will use general relativity and Maxwell's theory. Consequently, we are interested in deriving and analyzing exact solutions of the Einstein-Maxwell equations. On the other hand, the study of axially symmetric solutions of the Einstein and Einstein-Maxwell field equations corresponding to shells and disk-like configurations of matter, apart from its astrophysical relevance, has a clear purely mathematical interest.

Exact solutions that have relativistic static thin disks as their sources were first studied by Bonnor and Sackfield [1] and Morgan and Morgan [2, 3]. Subsequently, several classes of exact solutions corresponding to static [4 13] and stationary [14 18] thin disks have been obtained by different authors. The superposition of a static or stationary thin disk with a black hole has been considered in [19 27]. Thin disks around static black holes in a magnetic field have been studied in 28]. Relativistic disks embedded in an expanding Friedman-Lemaitre-Roberton-Walker universe have been studied in [29], and perfect fluid disks with halos in [30]. Furthermore, the stability of thin disks models has been investigated using a first order perturbation of the energy-momentum tensor in [31]. On the other hand, thin disks have been discussed as sources for Kerr-Newman fields [32, 33], magnetostatic axisymmetric fields [34, 35], and conformastatic and conformastationary metrics [36 38]. Also, models of electrovacuum static counterrotating dust disks were presented in [39], charged perfect fluid disks were studied in [40], and charged perfect fluid disks as sources of static and Taub-NUT-type spacetimes in [41, 42]. Also, monopole and dipole layers in curved spacetimes were analyzed in [43], and electromagnetic sources distributed on shells in a Schwarzschild background in [44].

Now, the thin disks with magnetic fields presented in [32 34 were obtained by means of the well-known "displace, cut and reflect" method that introduces a discontinuity in the first-order derivative of an otherwise smooth solution. The result is a solution with a singularity of the delta-function type in the entire $z=0$ hypersurface, and so it can be interpreted as an infinite thin disk. On the other hand, solutions that can be interpreted as thin disks of finite extension can be obtained if an appropriate coordinate system is introduced. A coordinate system that adapts naturally to a finite source and presents the required discontinuous behavior is given by the oblate spheroidal coordinates. Some examples of finite thin disks obtained from vacuum solutions expressed in these coordinates can be found in references [1, 2, 4, 7], and from electrovacuum solutions in reference [38].

In a previous work [38], we presented an infinite family of conformastatic axially symmetric charged dust disks of finite extension with well-behaved surface energy and charge densities. These disks have a charge density that is equal, up to a sign, to their energy density, and so they are examples of the commonly named "electrically counterpoised dust" equilibrium configuration. The energy density of the disks is everywhere positive and well-behaved, vanishing at the edge. Furthermore, since the energy density of the disks is everywhere positive and the disks are made of dust, all the models are in a complete agreement with all the energy conditions, a fact of particular relevance in the study of relativistic thin disks models. In the present paper, we extend these studies to obtain a model corresponding to a system that is composed of a thin disk and an exterior halo. The main purpose of this work is, then, to extend the previous electric field to include an electromagnetic field, and the previous "isolated" thin disk to include a thin disk-halo system.

In this work, we present a relativistic model describing a thin disk surrounded by a halo in presence of an electromagnetic field in a conformastatic spacetime [45 47]. Note that we take the definition in [46] as standard, following the original terminology by Synge [45]: conformastationary are those stationary spacetimes with a conformally flat space of orbits and the conformastatic comprise the static subset. The model is obtained by solving the Einstein-Maxwell equations and by using the distributional approach under the assumption that the energy-momentum tensor can be expressed as the sum of two distributional contributions, one due to the electromagnetic part and the other one due to a "material" part. In this way, explicit expressions for the energy, pressure, electric current and electromagnetic field are obtained for the disk region and for the halo region. In order to obtain the solutions, an auxiliary function is introduced that determines the functional dependence of the metric and the electromagnetic potential. It is also assumed that the auxiliary function depends explicitly on an additional function which is taken as a solution of the Laplace equation. A simple thin disk-halo model is obtained from the Kuzmin solutions of the Laplace equation. The energy-momentum tensor of the system agrees with all the energy conditions.

The plan of our paper is as follows. First, in Section II] the conformastatic line element is considered. The procedure to obtain electromagnetostatic, axially symmetric, relativistic thin disks surrounded by a material halo is also summarized in this section. Section III introduces a functional relationship dependence between the metric and 
electromagnetic potentials and an auxiliary function in order to obtain a family of solutions of the Einstein-Maxwell equations in terms of convenient solutions of the Laplace equations, modeling relativistic thin disk-halo systems. Next, the eigenvalue problem for disk with halos is studied and a particular model of a disk with halo is obtained from the Kuzmin solutions of the Laplace equation. In Section IIC the quadratic Riemann invariants and the electromagnetic invariants are studied, and the behavior of the Kuzmin disk with halo is analyzed. Finally, Section IV] is devoted to a discussion of the results.

\section{THE EINSTEIN-MAXWELL EQUATIONS AND THE THIN-DISK-HALO SYSTEM}

In order to formulate the Einstein-Maxwell equations for conformastatic axially symmetric spacetimes corresponding to an electromagnetized system, constituted by a thin disk and a halo surrounding the exterior of the disk, we first introduce coordinates $x^{a}=(t, \varphi, r, z)$ in which the metric tensor and the electromagnetic potential only depend on $r$ and $z$. We assume that these coordinates are quasicylindrical in the sense that the coordinate $r$ vanishes on the axis of symmetry and, for fixed $z$, increases monotonically to infinity, while the coordinate $z$, for $r$ fixed, increases monotonically in the interval $(-\infty, \infty)$. The azimuthal angle $\varphi$ ranges in the interval [0, 2 $\pi)$, as usual [2, 3]. We assume that there exists an infinitesimally thin disk, located at the hypersurface $z=0$, so that the components of the metric tensor $g_{a b}$ and the components of the electromagnetic potential $A_{a}$ are symmetrical functions of $z$ and their first derivatives have a finite discontinuity at $z=0$, accordingly,

$$
g_{a b}(r, z)=g_{a b}(r,-z), \quad A_{a}(r, z)=A_{a}(r,-z)
$$

in such a way that, for $z \neq 0$,

$$
g_{a b, z}(r, z)=-g_{a b, z}(r,-z), \quad A_{a, z}(r, z)=-A_{a, z}(r,-z) .
$$

The metric tensor and the electromagnetic potential are continuous at $z=0$,

$$
\begin{aligned}
& {\left[g_{a b}\right]=\left.g_{a b}\right|_{z=0^{+}}-\left.g_{a b}\right|_{z=0^{-}}=0,} \\
& {\left[A_{a}\right]=\left.A_{a}\right|_{z=0^{+}}-\left.A_{a}\right|_{z=0^{-}}=0,}
\end{aligned}
$$

whereas the discontinuity in the derivatives of the metric tensor and the electromagnetic potential can be written, respectively, as

$$
\begin{aligned}
\gamma_{a b} & =\left[g_{a b, z}\right], \\
\zeta_{a} & =\left[A_{a, z}\right],
\end{aligned}
$$

where the reflection symmetry with respect to $z=0$ has been used. Then, by using the distributional approach [48 50] or the junction conditions on the extrinsic curvature of thin shell [51 53], we can write the metric and the electromagnetic potential as

$$
\begin{aligned}
& g_{a b}=g_{a b}^{+} \theta(z)+g_{a b}^{-}\{1-\theta(z)\} \\
& A_{a}=A_{a}^{+} \theta(z)+A_{a}^{-}\{1-\theta(z)\},
\end{aligned}
$$

and thus the Ricci tensor reads

$$
R_{a b}=R_{a b}^{+} \theta(z)+R_{a b}^{-}\{1-\theta(z)\}+H_{a b} \delta(z)
$$

where $\theta(z)$ and $\delta(z)$ are, respectively, the Heaveside and Dirac distributions with support on $z=0$. Here $g_{a b}^{ \pm}$and $R_{a b}^{ \pm}$ are the metric tensors and the Ricci tensors of the $z \geq 0$ and $z \leq 0$ regions, respectively, and

$$
H_{a b}=\frac{1}{2}\left\{\gamma_{a}^{z} \delta_{b}^{z}+\gamma_{b}^{z} \delta_{a}^{z}-\gamma_{c}^{c} \delta_{a}^{z} \delta_{b}^{z}-g^{z z} \gamma_{a b}\right\}
$$

where all the quantities are evaluated at $z=0^{+}$. In agreement with Eq.(6), the energy-momentum tensor and the electric current density can be expressed as

$$
\begin{gathered}
T_{a b}=T_{a b}^{+} \theta(z)+T_{a b}^{-}\{1-\theta(z)\}+Q_{a b} \delta(z), \\
J_{a}=J_{a}^{+} \theta(z)+J_{a}^{-}\{1-\theta(z)\}+I_{a} \delta(z),
\end{gathered}
$$


where $T_{a b}^{ \pm}$and $J_{a b}^{ \pm}$are the energy-momentum tensors and electric current density of the $z \geq 0$ and $z \leq 0$ regions, respectively. Moreover, $Q_{a b}$ and $I_{a}$ represent the part of the energy-momentum tensor and the electric current density corresponding to the disk-like source.

To describe the physical properties of an electromagnetized system constituted by a thin disk surrounded by an exterior halo, $T_{a b}^{ \pm}$in (8a) can be written as

$$
T_{a b}^{ \pm}=E_{a b}^{ \pm}+M_{a b}^{ \pm},
$$

where $E_{a b}^{ \pm}$is the electomagnetic energy-momentum tensor

$$
E_{a b}=F_{a c} F_{b}{ }^{c}-\frac{1}{4} g_{a b} F_{c d} F^{c d},
$$

with $F_{a b}=A_{b, a}-A_{a, b}$ and $M_{a b}^{ \pm}$is an unknown "material" energy-momentum tensor (MEMT) to be obtained. Accordingly, the Einstein-Maxwell equations, in geometrized units such that $c=8 \pi G=\mu_{0}=\epsilon_{0}=1$, are equivalent to the system of equations

$$
\begin{aligned}
G_{a b}^{ \pm}=R_{a b}^{ \pm}-\frac{1}{2} g_{a b} R^{ \pm} & =E_{a b}^{ \pm}+M_{a b}^{ \pm}, \\
H_{a b}-\frac{1}{2} g_{a b} H & =Q_{a b}, \\
\hat{F}_{ \pm}^{a b}, b & =\hat{J}_{ \pm}^{a} \\
{\left[\hat{F}^{a b}\right] n_{b} } & =\hat{I}^{a}
\end{aligned}
$$

where $H=g^{a b} H_{a b}$ and $\hat{a}=\sqrt{-g} a$, being $g$ the determinant of the metric tensor. Here, "[ ]" in the expressions $\left[\hat{F}^{a b}\right]$ denotes the jump of $\hat{F}^{a b}$ across of the surface $z=0$ and $n_{b}$ denotes an unitary vector in the direction normal to it.

Now, in order to obtain explicit forms for the Einstein-Maxwell equations corresponding to the electromagnetized disk-halo system, we take the metric tensor as given by the conformastatic line element [45, 46]

$$
\mathrm{d} s^{2}=-\mathrm{e}^{2 \phi} \mathrm{d} t^{2}+\mathrm{e}^{2 \psi}\left[r^{2} \mathrm{~d} \varphi^{2}+\mathrm{d} r^{2}+\mathrm{d} z^{2}\right],
$$

where the metric functions $\phi$ and $\psi$ depend only on $r$ and $z$, and the electromagnetic potential is

$$
A_{\alpha}=\left(A_{0}, A, 0,0\right) .
$$

We also assume that the electric potential $A_{0}$ and the magnetic potential $A$ are independent of $t$. For later use, the corresponding field equations and the components of the energy-momentum tensor are given explicitly in the Appendix.

Furthermore, from Eq.113) we have for the current density in these regions,

$$
\begin{aligned}
& \hat{J}_{ \pm}^{0}=-r e^{\psi-\phi}\left\{\nabla^{2} A_{0}-\nabla(\phi-\psi) \cdot \nabla A_{0}\right\}, \\
& \hat{J}_{ \pm}^{1}=-r^{-1} e^{\phi-\psi}\left\{\nabla^{2} A+\nabla(\phi-\psi) \cdot \nabla A-\frac{2}{r} A_{, r}\right\},
\end{aligned}
$$

where, as we know, $\hat{J}_{ \pm}^{a}=J_{ \pm}^{a} \sqrt{-g}$. The "true" surface energy-momentum tensor of the disk, $S_{a b}$, can be obtained through the relation

$$
S_{a b}=\int Q_{a b} \delta(z) d s_{n}=\sqrt{g_{z z}} Q_{a b}
$$

where $d s_{n}=\sqrt{g_{z z}} d z$ is the "physical measure" of length in the direction normal to the $z=0$ plane. Accordingly, for the metric (15), the nonzero components of $S_{a b}$ are given by

$$
\begin{aligned}
& S_{0}^{0}=4 e^{-\psi} \psi_{, z}, \\
& S_{1}^{1}=2 e^{-\psi}(\phi+\psi)_{, z}, \\
& S_{2}^{2}=2 e^{-\psi}(\phi+\psi)_{, z},
\end{aligned}
$$

where all the quantities are evaluated at $z=0^{+}$. The "true" current density on the surface of the disk, $\mathcal{J}_{a}$, can be obtained through the relation

$$
\mathcal{J}^{a}=\int I^{a} \delta(z) \sqrt{g_{z z}} d z
$$


or explicitly

$$
\begin{aligned}
\mathcal{J}^{0} & =e^{-(\psi+2 \phi)}\left[A_{0, z}\right], \\
\mathcal{J}^{1} & =-r^{-2} e^{-3 \psi}\left[A_{, z}\right],
\end{aligned}
$$

where all the quantities are evaluated on the surface of the disk, and $\left[A_{a, z}\right]$ denotes the jump of the derivative of $A_{a}$ across the surface $z=0$. Now, in order to analyze the physical characteristics of the system it is convenient to express the energy-momentum tensor $T_{a b}$ and the electric current density in terms of an orthonormal tetrad. We will use the tetrad of the "locally static observers" (LSO) [37], i.e., observers at rest with respect to infinity, which is given by

$$
e_{(b)}^{a}=\left\{V^{a}, W^{a}, X^{a}, Y^{a}\right\}
$$

where

$$
\begin{aligned}
V^{a} & =e_{(0)}^{a}=e^{-\phi} \delta_{0}^{a}, \\
W^{a} & =e_{(1)}^{a}=r^{-1} e^{-\psi} \delta_{1}^{a}, \\
X^{a} & =e_{(2)}^{a}=e^{-\psi} \delta_{2}^{a}, \\
Y^{a} & =e_{(3)}^{a}=e^{-\psi} \delta_{3}^{a} .
\end{aligned}
$$

In terms of this tetrad $M_{a b}^{ \pm}$and $\hat{J}_{a}^{ \pm}$can be expressed as

$$
\begin{aligned}
M_{ \pm}^{a b} & =M_{(0)(0)}^{ \pm} V^{a} V^{b}+M_{(1)(1)}^{ \pm} W^{a} W^{b}+M_{(2)(2)}^{ \pm} X^{a} X^{b}+M_{(3)(3)}^{ \pm} Y^{a} Y^{b} \\
& -M_{(0)(1)}^{ \pm}\left\{V^{a} W^{b}+W^{a} V^{b}\right\}+M_{(2)(3)}^{ \pm}\left\{X^{a} Y^{b}+Y^{a} X^{b}\right\}, \\
\hat{J}_{ \pm}^{a} & =-\hat{J}_{(0)}^{ \pm} V^{a}+\hat{J}_{(1)}^{ \pm} W^{a} .
\end{aligned}
$$

The explicit expressions for these quantities are given in the Appendix.

In the same way, by using the LSO tetrad, the surface energy-momentum tensor and the surface current density of the disk as well as the electric current on the disk can be written in the canonical form as

$$
\begin{aligned}
S^{a b} & =S_{(0)(0)} V^{a} V^{b}+S_{(1)(1)} W^{a} W^{b}+S_{(2)(2)} X^{a} X^{b} \\
\mathcal{J}^{a} & =-\mathcal{J}_{(0)} V^{a}+\mathcal{J}_{(1)} W^{a}
\end{aligned}
$$

with

$$
\begin{aligned}
S_{(0)(0)} & =-4 e^{-\psi} \psi, z, \\
S_{(1)(1)} & =2 e^{-\psi}(\phi+\psi)_{, z}=S_{(2)(2)}, \\
\mathcal{J}_{(0)} & =-e^{-(\psi+\phi)}\left[A_{0, z}\right], \\
\mathcal{J}_{(1)} & =-r^{-1} e^{-2 \psi}[A, z],
\end{aligned}
$$

where we have used (19) and (20) and again all the quantities are evaluated at $z=0^{+}$.

\section{THIN DISK WITH AN ELECTROMAGNETIZED MATERIAL HALO}

In the precedent section, we discussed a generalized formalism in which conformastatic axially symmetric solutions of the Einstein-Maxwell can be interpreted in terms of a thin disk placed at the surface $z=0$ surrounded by a distribution of electrically charged matter located in the $z \geq 0$ and $z \leq 0$ regions, whose physical properties can be studied by analyzing the behavior of $S_{(a)(b)}, \mathcal{J}_{(a)}, M_{(a)(b)}^{ \pm}$and $J_{(a)}^{ \pm}$. To this end, it is necessary to "choose" a convenient explicit form for the metric. It turns out that the assumption $\psi=-\phi$ leads to a considerable simplification of the problem. Indeed, the equations (A6) reduce to 


$$
\begin{aligned}
M_{(0)(0)}^{ \pm} & =\frac{1}{4} f^{-1}\left\{4 f \nabla^{2} f-5 \nabla f \cdot \nabla f-2 f \nabla A_{0} \cdot \nabla A_{0}-2 r^{-2} f^{3} \nabla A \cdot \nabla A\right\}, \\
M_{(0)(1)}^{ \pm} & =-r^{-1} f \nabla A_{0} \cdot \nabla A, \\
M_{(1)(1)}^{ \pm} & =\frac{1}{4} f^{-1}\left\{\nabla f \cdot \nabla f-2 f \nabla A_{0} \cdot \nabla A_{0}-2 r^{-2} f^{3} \nabla A \cdot \nabla A\right\}, \\
M_{(2)(2)}^{ \pm} & =\frac{1}{4} f^{-1}\left\{-\left(f_{, r}^{2}-f_{, z}^{2}\right)+2 f\left(A_{0, r}^{2}-A_{0, z}^{2}\right)-2 r^{-2} f^{3}\left(A_{, r}^{2}-A_{, z}^{2}\right)\right\}, \\
M_{(3)(3)}^{ \pm} & =-M_{(2)(2)}^{ \pm}, \\
M_{(2)(3)}^{ \pm} & =-\frac{1}{2} f^{-1} f_{, r} f_{, z}+A_{0, r} A_{0, z}-r^{-2} f^{2} A_{, r} A_{, z}, \\
\hat{J}_{(0)}^{ \pm} & =r f^{1 / 2} \nabla \cdot\left(f^{-1} \nabla A_{0}\right), \\
\hat{J}_{(1)}^{ \pm} & =-r^{2} f^{-1 / 2} \nabla \cdot\left(r^{-2} f \nabla A\right) .
\end{aligned}
$$

In the same way, for the nonzero components of the energy-momentum and the current density on the surface of the disk (26) we have, respectively,

$$
S_{(0)(0)}=4\left(f^{1 / 2}\right)_{, z},
$$

and

$$
\begin{aligned}
& \mathcal{J}_{(0)}=-\left[A_{0, z}\right], \\
& \mathcal{J}_{(1)}=-r^{-1} f\left[A_{, z}\right],
\end{aligned}
$$

where "[ ]" denotes the jump across of the disk, $f \equiv e^{2 \phi}$ and all the quantities are evaluated on the surface of the disk. We will suppose that there is no electric current in the halo, i. e., we assume that $\hat{J}_{(\alpha)}^{ \pm} \equiv 0$. Then, if $\hat{\mathbf{e}}_{\varphi}$ is a unit vector in the azimuthal direction and $\lambda$ is any reasonable function independent of the azimuth, one has the identity

$$
\nabla \cdot\left(r^{-1} \hat{\mathbf{e}}_{\varphi} \times \nabla \lambda\right)=0 .
$$

Equation (27h) may be regarded as the integrability condition for the existence of the function $\lambda$ defined by

$$
r^{-2} f \nabla A=r^{-1} \hat{\mathbf{e}}_{\varphi} \times \nabla \lambda,
$$

or, equivalently

$$
-f^{-1} \nabla \lambda=r^{-1} \hat{\mathbf{e}}_{\varphi} \times \nabla A .
$$

Hence, the identity (30) implies the equation

$$
\nabla \cdot\left(f^{-1} \nabla \lambda\right)=0
$$

for the new "potential" $\lambda(r, z)$. In order to obtain an explicit form of the metric and electromagnetic potential, we suppose that $f, A_{0}$ and $A$ depend explicitly on $\lambda$. Then, we obtain from 27g]

$$
\left(-f^{-1} f^{\prime} A_{0}^{\prime}+A_{0}^{\prime \prime}\right) \nabla \lambda \cdot \nabla \lambda+A_{0}^{\prime} \nabla^{2} \lambda=0
$$

where ()$^{\prime}$ denotes derivatives respect to $\lambda$. Whereas, from (33), we have

$$
-f^{-1} f^{\prime} \nabla \lambda \cdot \nabla \lambda+\nabla^{2} \lambda=0 .
$$

Consequently, by inserting (35) into (34), we obtain $A_{0}^{\prime \prime}=0$, whose general solution is $A_{0}=k_{1} \lambda+k_{2}$, where $k_{1}$ and $k_{2}$ are constants. We now proceed to determine the function $\lambda(r, z)$. Let us assume in (35) the very useful simplification $f^{\prime} f^{-1}=k$, where $k$ is an arbitrary constant. Then, $f=k_{3} e^{k \lambda}$, and

$$
\nabla^{2} \lambda=k \nabla \lambda \cdot \nabla \lambda
$$

where $k_{3}$ is a constant. Furthermore, if we now assume the existence of a function $U=k_{4} e^{-k \lambda}+k_{5}$, with $k_{4}$ and $k_{5}$ being arbitrary constants, then

$$
\nabla^{2} U=-k k_{4} e^{-k \lambda}\left(\nabla^{2} \lambda-k \nabla \lambda \cdot \nabla \lambda\right)=0
$$


and, consequently, $\lambda$ can be represented in terms of solutions of the Laplace equation:

$$
e^{k \lambda}=\frac{k_{4}}{U-k_{5}} ; \quad \nabla^{2} U=0 .
$$

Then, in order to have an asymptotically flat spacetime at infinity, we will only consider functions $U$ that vanish at infinity and we must take $k_{5}=-k_{3} k_{4}$ in Eq. (38).

On the other hand, from Eq.(31) we obtain the following relationship between $A$ and $\lambda$ :

$$
\nabla A=A_{, r} \hat{\mathbf{e}}_{r}+A_{, z} \hat{\mathbf{e}}_{z}=r f^{-1} \hat{\mathbf{e}}_{\varphi} \times\left(\lambda_{, r} \hat{\mathbf{e}}_{r}+\lambda_{, z} \hat{\mathbf{e}}_{z}\right),
$$

that is, $A_{, r}=-r f^{-1} \lambda_{, z}$, and $A_{, z}=r f^{-1} \lambda_{, r}$, or, in terms of $U, A_{, r}=k_{6} r U_{, z}$, and $A_{, z}=-k_{6} r U_{, r}$, where $k_{6}=$ $1 /\left(k k_{3} k_{4}\right)$. Then, using this solution for the nonzero components of $M_{(a)(b)}^{ \pm}$, we have from (27):

$$
\begin{aligned}
M_{(0)(0)}^{ \pm} & =\frac{U_{, r}^{2}+U_{, z}^{2}}{4\left(U-k_{5}\right)^{2}}\left\{3 f-k_{7}\right\}, \\
M_{(1)(1)}^{ \pm} & =\frac{U_{, r}^{2}+U_{, z}^{2}}{4\left(U-k_{5}\right)^{2}}\left\{f-k_{7}\right\}, \\
M_{(2)(2)}^{ \pm} & =-\frac{U_{, r}^{2}-U_{, z}^{2}}{4\left(U-k_{5}\right)^{2}}\left\{f-k_{7}\right\}, \\
M_{(2)(3)}^{ \pm} & =-\frac{U_{, r} U_{, z}}{2\left(U-k_{5}\right)^{2}}\left\{f-k_{7}\right\}, \\
M_{(3)(3)}^{ \pm} & =-M_{(2)(2)}^{ \pm} .
\end{aligned}
$$

Furthermore, for the nonzero components of the energy-momentum tensor and the current density on the surface of the disk we have from (28) and (29) respectively,

$$
S_{(0)(0)}=2\left(k_{3} k_{4}\right)^{1 / 2} \frac{U_{, z}}{\left(U-k_{5}\right)^{3 / 2}}
$$

and

$$
\begin{aligned}
& \mathcal{J}_{(0)}=\frac{k_{1}}{k}\left[\frac{U_{, z}}{U-k_{5}}\right], \\
& \mathcal{J}_{(1)}=\frac{\left[U_{, r}\right]}{k\left(U-k_{5}\right)},
\end{aligned}
$$

with

$$
f=\frac{k_{3} k_{4}}{U-k_{5}} \quad \text { and } \quad k_{7}=\frac{2\left(k_{1}^{2}+1\right)}{k^{2}}
$$

As we can see, $S_{(0)(0)}$ is the only nonzero component of the surface energy-momentum tensor; we can then interpret it as the surface energy density of the disk $\epsilon(r)$ as seen from any LSO. So, in order that the surface energy-momentum tensor will be in agreement with the energy conditions, we only need to require that $\left.U_{, z}\right|_{0^{+}} \geq 0$ and take $k_{3} k_{4}>0$. Likewise, we interpret $\sigma(r)=\mathcal{J}_{(0)}$ and $\mathcal{I}=\mathcal{J}_{(1)}$ as the charge density and electric current density on the surface of the disk, respectively.

\section{A. The eigenvalue problem for the energy-momentum tensor of the halo}

When the tensor $M_{(a)(b)}^{ \pm}$in the LSO tetrad is diagonal, its interpretation is immediate. In our case, however, $M_{(2)(3)}^{ \pm} \neq 0$ and, therefore, it is necessary to rewrite $M_{(a)(b)}^{ \pm}$in the canonical form. To this end, we must solve the eigenvalue problem for $M_{(a)(b)}^{ \pm}$, i.e.,

$$
M_{(a)(b)}^{ \pm} \xi_{A}^{(b)}=\lambda_{A} \eta_{(a)(b)} \xi_{A}^{(b)}
$$


and express the physically relevant quantities in terms of the eigenvalues and eigenvectors. The solution of the eigenvalue problem in the LSO orthonormal tetrad leads to the eigenvalues

$$
\begin{aligned}
\lambda_{0} & =-M_{(0)(0)}^{ \pm}, \\
\lambda_{1} & =M_{(1)(1)}^{ \pm}, \\
\lambda_{ \pm} & = \pm \sqrt{D}
\end{aligned}
$$

and the corresponding eigenvectors are given by

$$
\begin{aligned}
& \xi_{0}^{(a)}=V^{(a)}=(1,0,0,0), \\
& \xi^{(a)}{ }_{1}=X^{(a)}=(0,1,0,0), \\
& \xi^{(a)}{ }_{+}=Y^{(a)}=N(0,0,1,-\omega), \\
& \xi^{(a)}{ }_{-}=Z^{(a)}=N(0,0, \omega, 1),
\end{aligned}
$$

where

$$
\begin{aligned}
D & =\left(M_{(2)(2)}^{ \pm}\right)^{2}+\left(M_{(2)(3)}^{ \pm}\right)^{2}, \\
\omega & =\frac{M_{(2)(2)}^{ \pm}-\sqrt{D}}{M_{(2)(3)}^{ \pm}}, \\
N & =\frac{1}{\sqrt{1+\omega^{2}}} .
\end{aligned}
$$

In terms of the tetrad $\xi^{(a)}{ }_{A}=\left\{V^{(a)}, X^{(a)}, Y^{(a)}, Z^{(a)}\right\}$, the tensor $M_{(a)(b)}^{ \pm}$can be written in the canonical form

$$
M_{(a)(b)}^{ \pm}=\varepsilon V_{(a)} V_{(b)}+p_{1} X_{(a)} X_{(b)}+p_{2} Y_{(a)} Y_{(b)}+p_{3} Z_{(a)} Z_{(b)} .
$$

Consequently, we can interpret $\varepsilon$ as the energy density of the halo and $p_{1}, p_{2}$ and $p_{3}$ as the pressure in the principal directions of the halo. So, we have that the energy density of the halo is given by

$$
\varepsilon=M_{(0)(0)}^{ \pm}
$$

whereas for the principal pressure we have

$$
p=p_{1}=p_{2}=-p_{3}=M_{(1)(1)}^{ \pm},
$$

and

$$
\langle p\rangle=\frac{p_{1}+p_{2}+p_{3}}{3}=\frac{p}{3}
$$

is the average value of the pressure.

Accordingly, we have an anisotropic fluid with a non-barotropic equation of state, which can be written as

$$
p=p(f, \varepsilon)=\left[\frac{f-k_{7}}{3 f-k_{7}}\right] \varepsilon
$$

in such a way that the pressure not only depends on the energy density but also on the gravitational and electromagnetic fields through the function $f$. Now, it is easy to see that, in order that the material energy-momentum tensor satisfy all the energy conditions, the function $U$ must be constrained by

$$
U \leq \frac{\left(2-k_{7}\right) k_{3} k_{4}}{k_{7}},
$$

where we used the constrain on $k_{5}$ in Eq. (38) needed to have an asymptotically flat spacetime at infinity. 


\section{B. The Kuzmin-like solution}

One can find many different models for a relativistic thin disk surrounded by a material electromagnetized halo by choosing different kind of solutions $U$ of the Laplace equation. Let us consider the particular case of Kuzmin's solution [54, 55]

$$
U=-\frac{m}{\sqrt{r^{2}+(|z|+a)^{2}}}, \quad(a, m>0) .
$$

At points with $z<0, U$ is identical to the potential of a point mass $m$ located at the point $(r, z)=(0,-a)$, and when $z>0, U$ coincides with the potential generated by a point mass at $(0, a)$. Hence $\nabla^{2} U$ must vanish everywhere except on the plane $z=0$. By applying Gauss theorem to a flat volume that contains a small portion of the plane $z=0$, we conclude that $U$ is generated by the surface density of a Newtonian mass

$$
\rho(r, z=0)=\frac{a m}{2 \pi\left(r^{2}+a^{2}\right)^{3 / 2}} .
$$

Furthermore, without loss of generality we can choose the constants $k_{3}=k_{4}=1$. Then, for the metric potential we have

$$
e^{2 \phi}=\frac{\sqrt{\tilde{r}^{2}+(|\tilde{z}|+1)^{2}}}{\sqrt{\tilde{r}^{2}+(|\tilde{z}|+1)^{2}}-\tilde{m}},
$$

where we introduced the dimensionless variables $\tilde{r}=r / a, \tilde{z}=z / a$, and $\tilde{m}=m / a$. With this metric potential it is straightforward to calculate the dimensionless radial and axial components of the electric field, $\tilde{E}_{r}=a E_{r}$ and $\tilde{E}_{z}=a^{2} E_{z}$, and the dimensionless radial and axial components of the magnetic field, $\tilde{B}_{r}=B_{r}$ and $\tilde{B}_{z}=B_{z}$. We obtain

$$
\begin{aligned}
& \tilde{E}_{r}=-\frac{k_{1} \tilde{m} \tilde{r}}{k\left\{\tilde{r}^{2}+(|\tilde{z}|+1)^{2}\right\}\left\{\sqrt{\tilde{r}^{2}+(|\tilde{z}|+1)^{2}}-\tilde{m}\right\}}, \\
& \tilde{E}_{z}=-\frac{k_{1} \tilde{m}|\tilde{z}|(\tilde{z}+1)}{k \tilde{z}\left\{\tilde{r}^{2}+(|\tilde{z}|+1)^{2}\right\}\left\{\sqrt{\tilde{r}^{2}+(|\tilde{z}|+1)^{2}}-\tilde{m}\right\}}, \\
& \tilde{B}_{r}=-\frac{\tilde{m} \tilde{r}^{2}}{k\left\{\tilde{r}^{2}+(|\tilde{z}|+1)^{2}\right\}^{3 / 2}}, \\
& \tilde{B}_{z}=-\frac{\tilde{m} \tilde{r}|\tilde{z}|(\tilde{z}+1)}{k \tilde{z}\left\{\tilde{r}^{2}+(|\tilde{z}|+1)^{2}\right\}^{3 / 2}},
\end{aligned}
$$

were we have used the definitions $E_{r}=A_{0, r}, E_{z}=A_{0, z}, B_{r}=A_{, z}$ and $B_{z}=-A_{, r}$.

Substituting (53) into (41) and (42b), we obtain for the dimensionless energy and charge density on surface of the $\operatorname{disk}, \tilde{\epsilon}=a \epsilon, \tilde{\sigma}=a \sigma$, respectively,

$$
\begin{aligned}
\tilde{\epsilon}(\tilde{r}) & =\frac{2 \tilde{m}}{\left(\tilde{r}^{2}+1\right)^{3 / 4}\left(\sqrt{\tilde{r}^{2}+1}-\tilde{m}\right)^{3 / 2}}, \\
\tilde{\sigma}(\tilde{r}) & =\frac{2 k_{1} \tilde{m}}{k\left(\tilde{r}^{2}+1\right)\left(\sqrt{\tilde{r}^{2}+1}-\tilde{m}\right)} .
\end{aligned}
$$

The substitution of (53) into (48) and (49) allows us to write the dimensionless energy of the halo $\tilde{\varepsilon}(\tilde{r}, \tilde{z})=a^{2} \varepsilon(r, z)$ and the dimensionless pressure of the halo $\tilde{p}(\tilde{r}, \tilde{z})=a^{2} p(r, z)$ as

$$
\begin{aligned}
& \tilde{\varepsilon}(\tilde{r}, \tilde{z})=\tilde{T}\left\{\frac{3 \sqrt{\tilde{r}^{2}+(|\tilde{z}|+1)^{2}}}{\sqrt{\tilde{r}^{2}+(|\tilde{z}|+1)^{2}}-\tilde{m}}-k_{7}\right\}, \\
& \tilde{p}(\tilde{r}, \tilde{z})=\tilde{T}\left\{\frac{\sqrt{\tilde{r}^{2}+(|\tilde{z}|+1)^{2}}}{\sqrt{\tilde{r}^{2}+(|\tilde{z}|+1)^{2}}-\tilde{m}}-k_{7}\right\},
\end{aligned}
$$

where

$$
\tilde{T}=\frac{\tilde{m}}{4\left\{\tilde{r}^{2}+(|\tilde{z}|+1)^{2}\right\}\left\{\sqrt{\tilde{r}^{2}+(|\tilde{z}|+1)^{2}}-\tilde{m}\right\}^{2}} .
$$


$\tilde{\epsilon}$

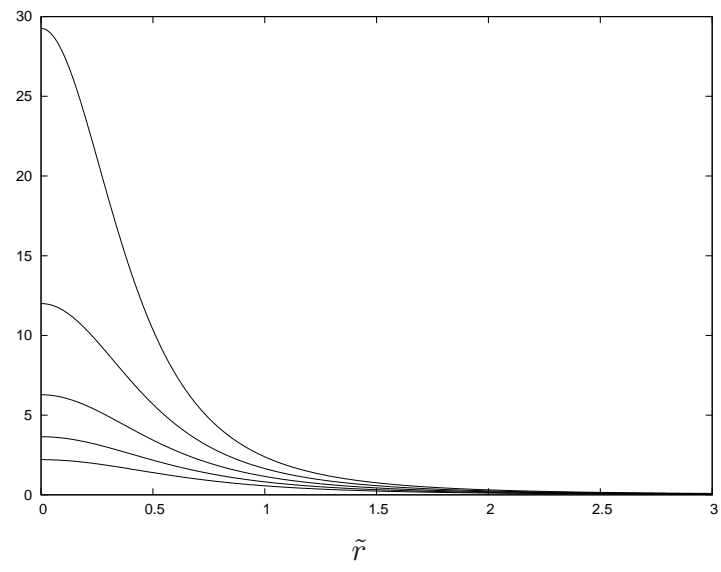

(a) $\tilde{\sigma}$

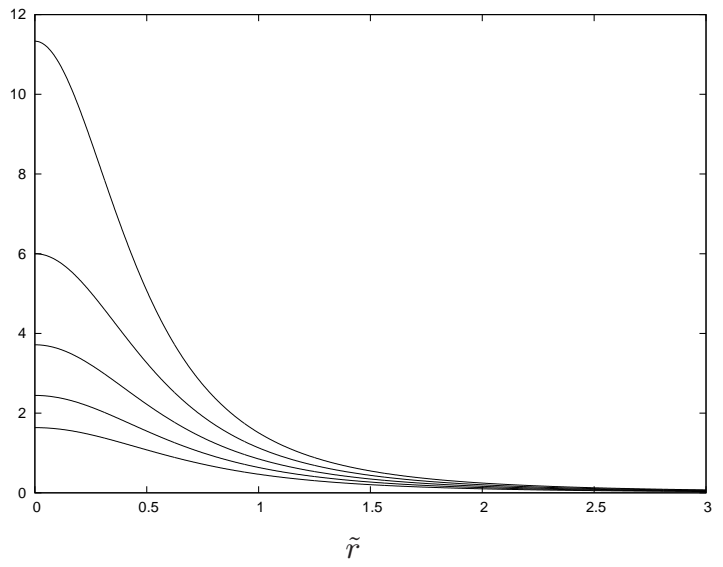

(b)

FIG. 1. Dimensionless surface energy $\tilde{\epsilon}$ and charge $\tilde{\sigma}$ densities as a function of $\tilde{r}$. In each case, we plot $\tilde{\epsilon}(\tilde{r})$ and $\tilde{\sigma}(\tilde{r})$ for different values of the parameter $\tilde{m}$. First, we take $\tilde{m}=0.45$ (the bottom curve in each plot) and then $0.55,0.75$ and $\tilde{m}=0.85$ (the top curve in each plot).

To fix the values of the constants that enter the solution, we consider the energy conditions at infinity, $\tilde{r}, \tilde{z} \longrightarrow \infty$, and at the center of the body, $\tilde{r}, \tilde{z} \longrightarrow 0$. Then, it can be shown that all the energy conditions are satisfied at these extreme regions if

$$
\tilde{m}<1
$$

for the disk and, additionally,

$$
\left.-\sqrt{k_{1}^{2}+1}<k<\sqrt{k_{1}^{2}+1} \quad \text { (that is } k_{7}<2\right)
$$

for the halo.

In Fig. 1(a), we show the dimensionless surface energy density on the disk $\tilde{\epsilon}$ as a function of $\tilde{r}$ and for different values of the parameter $\tilde{m}$. First, we take $\tilde{m}=0.45$ (the bottom curve in the plot) and then $0.55,0.75$ and $\tilde{m}=0.85$ (the top curve in the plot). It can be seen that the energy density is everywhere positive fulfilling the energy conditions. It can be observed that for all the values of $\tilde{m}$ the maximum of the energy density occurs at the center of the disk and that it vanishes sufficiently fast as $r$ increases. It can also be observed that the energy density in the central region of the disk increases as the values of the parameter $\tilde{m}$ increase. We have also plotted in Fig. 1(b) the charge density $\tilde{\sigma}$ as a function of $\tilde{r}$. In each case, we plot $\tilde{\sigma}$ for different values of the parameter $\tilde{m}$. We observe that the electric charge density has a behavior similar to that of the energy. This is consistent with the fact that the mass and the charge are more densely concentrated in the center of the disk. We also computed these functions for other values of the parameters $\tilde{m}$ in the interval $(0,1)$ and in all cases we found a similar behavior.

In Fig. 2(a) and in Fig. 2(b), we illustrate the behavior of the surfaces and level curves of the matter density in the halo around of the disk $(r \geq 0, z \geq 0)$ for the parameters $\tilde{m}=075$ and $k_{7}=1$. We can see that the energy is everywhere positive, its maximum occurs around the center of the disk, and it vanishes sufficiently fast as $r$ increases.

Notice that in the limit $m \rightarrow 0$, the gravitational and electromagnetic fields vanish identically, and the metric becomes flat. This is an important limiting case because it indicates that $m$ determines the mass of the disk and the halo, and that the electromagnetic field exists only in connection with the disk-halo configuration. Moreover, when $k_{1}=0$, we obtain $A_{0}=k_{2}=$ const., a "purely magnetic" solution. Then, $k_{1}$ determines the electric charge of the distribution whereas $k_{6}=1 / k$ must be associated with the magnetic field.

The energy condition $\tilde{m}<1$ or, equivalently, $m<a$ imposes a maximum on the value of the mass parameter. Recall that in the Kuzmin solution $a$ represents the distance along the axis between the equatorial plane and the point where the mass $m$ is situated; accordingly, $a$ can be interpreted as a parameter determining a proper length for the configuration. Then, the inequality $m / a<1$ represents a condition on the "specific mass" of the system. This resembles the well-known Chandrasekhar limit for a spherically symmetric mass distribution, stating that the condition mass/radius $<4 / 9$ must be satisfied in order to avoid gravitational collapse. The energy condition for the 


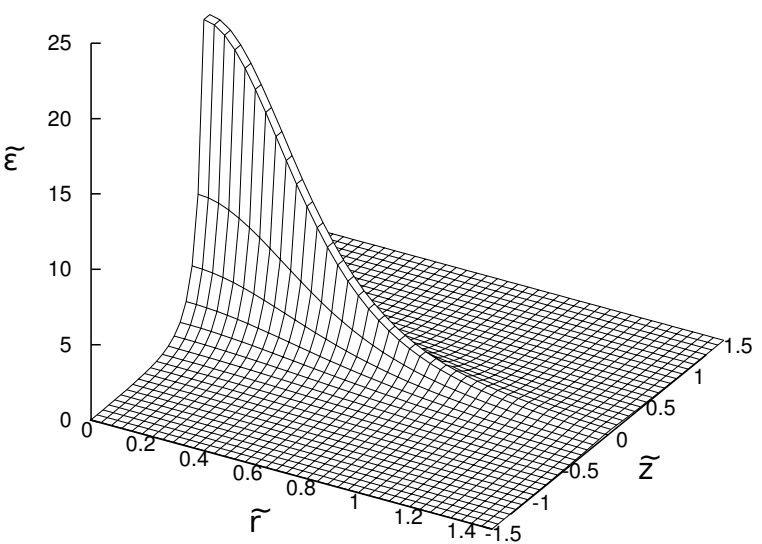

(a)

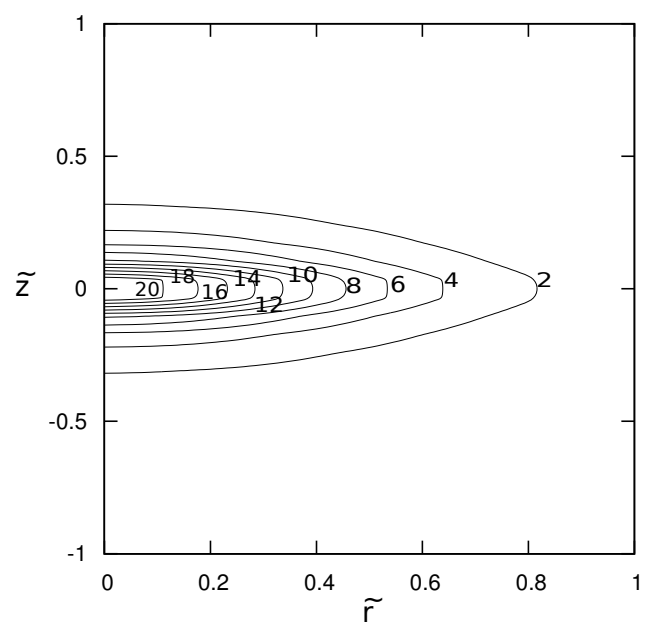

(b)

FIG. 2. Surface plot an level curves of the energy density $\tilde{\varepsilon}$ on the exterior halo as a function of $\tilde{r}$ and $\tilde{z}$ with parameters $\tilde{m}=0.75$ and $\tilde{k_{7}}=1$.

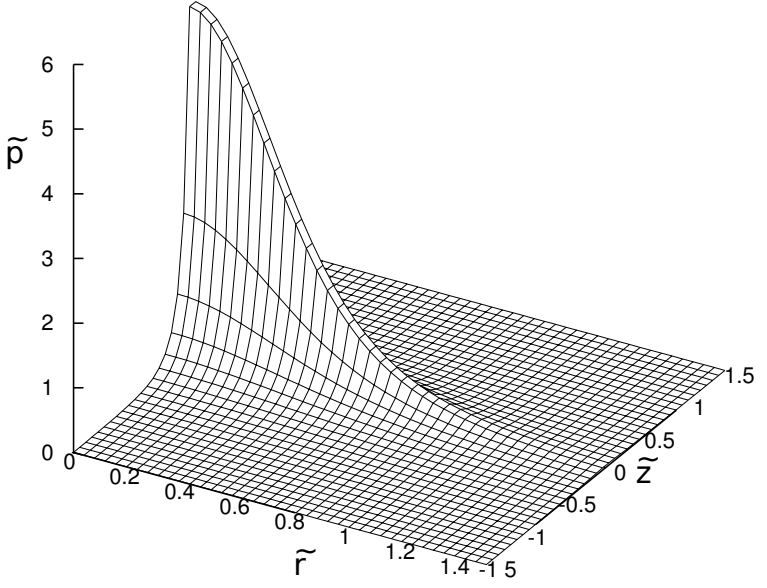

$(a)$

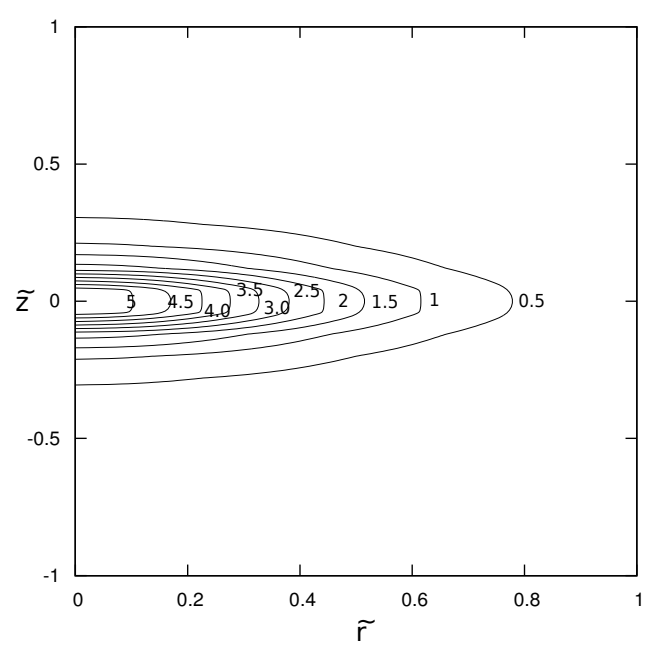

$(b)$

FIG. 3. Surface plot and level curves of the radial pressure $\tilde{p}$ on the exterior halo as a function of $\tilde{r}$ and $\tilde{z}$ with parameters $\tilde{m}=0.75$ and $\tilde{k_{7}}=1$.

halo $k^{2}<1+k_{1}^{2}$ also represents a relationship between the parameters that characterize the electric and magnetic properties of the system.

We conclude that all the relevant quantities show a physically reasonable behavior within the allowed range of values of the parameters. This indicates that the solution presented here can be used to describe the gravitational field of a static thin disk surrounded by a material halo with a non-trivial electromagnetic field.

\section{Singular behavior of the Kuzmin-like solution}

In order to study the singularities that could be present in the space described by the Kuzmin-like solution derived in the last subsection, we compute the most important quadratic curvature scalars, namely, the Kretschmann $\mathcal{K}_{I}$, the 


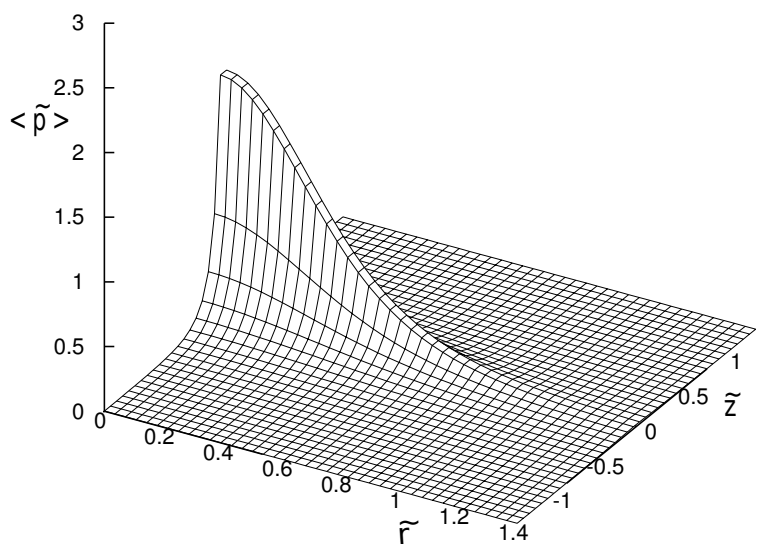

(a)

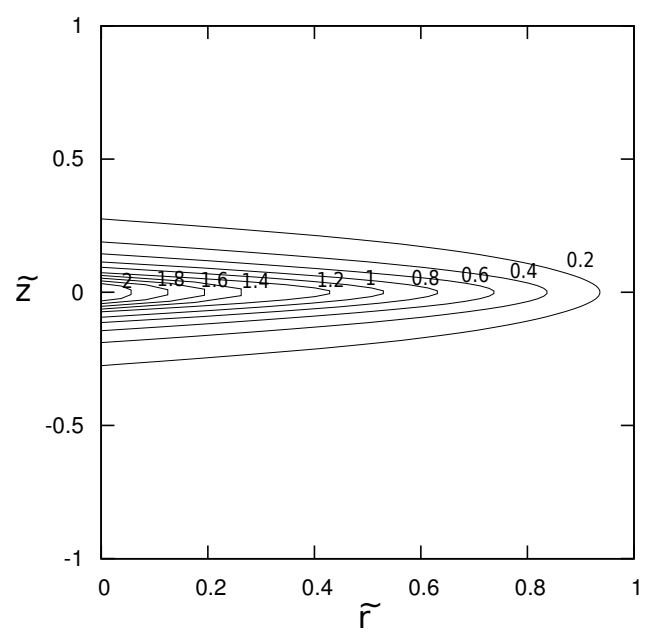

(b)

FIG. 4. Surface plot an level curves of the average value of the radial pressure $\langle\tilde{p}>$ on the exterior halo as a function of $\tilde{r}$ and $\tilde{z}$ with parameters $\tilde{m}=0.75$ and $\tilde{k_{7}}=1$.

Chern-Pontryagin $\mathcal{K}_{I I}$ and the Euler invariants $\mathcal{K}_{I I I}$ defined as $[56$ ]

$$
\begin{aligned}
\mathcal{K}_{I} & =R^{a b c d} R_{a b c d}, \\
\mathcal{K}_{I I} & =\left[{ }^{*} R\right]^{a b c d} R_{a b c d}=\frac{\epsilon^{a b}{ }_{i j} R^{i j c d} R_{a b c d}}{\sqrt{-g}}, \\
\mathcal{K}_{I I I} & =\left[{ }^{*} R^{*}\right]^{a b c d} R_{a b c d}=\frac{\epsilon^{a b i j} \epsilon^{c d k l} R_{i j k l} R_{a b c d}}{g} .
\end{aligned}
$$

As for the Maxwell field, we consider the electromagnetic invariants

$$
\begin{aligned}
\mathcal{F}_{I} & =F_{a b} F^{a b} \\
\mathcal{F}_{I I} & =F_{a b} F^{* a b} .
\end{aligned}
$$

Here $g=\operatorname{det}\left(g_{a b}\right), \epsilon^{a b c d}$ is the Levi-Civita symbol and the asterisk denotes the dual operation. By using the solution (55) we can cast these invariants as

$$
\begin{aligned}
\mathcal{K}_{I}(\tilde{r}, \tilde{z}) & =\frac{\tilde{m}^{2}\left\{48\left[(|\tilde{z}|+1)^{2}+\tilde{r}^{2}\right]-32 \tilde{m} \sqrt{(|\tilde{z}|+1)^{2}+\tilde{r}^{2}}+11 \tilde{m}^{2}\right\}}{4\left[(|\tilde{z}|+1)^{2}+\tilde{r}^{2}\right]\left[\sqrt{(|\tilde{z}|+1)^{2}+\tilde{r}^{2}}-\tilde{m}\right]^{6}}, \\
\mathcal{K}_{I I}(\tilde{r}, \tilde{z}) & =0, \\
\mathcal{K}_{I I I}(\tilde{r}, \tilde{z}) & =\frac{16 \tilde{m}^{2}\left\{3\left[(|\tilde{z}|+1)^{2}+\tilde{r}^{2}\right]-2 \tilde{m} \sqrt{(|\tilde{z}|+1)^{2}+\tilde{r}^{2}}\right\}}{\left[(|\tilde{z}|+1)^{2}+\tilde{r}^{2}\right]\left[\sqrt{(|\tilde{z}|+1)^{2}+\tilde{r}^{2}}-\tilde{m}\right]^{6}},
\end{aligned}
$$

whereas the electromagnetic invariants are

$$
\begin{aligned}
& \mathcal{F}_{I}=\frac{2\left(1-k_{1}^{2}\right) \tilde{m}^{2} a^{2}}{k^{2}\left[(|\tilde{z}|+1)^{2}+\tilde{r}^{2}\right]\left[\sqrt{(|\tilde{z}|+1)^{2}+\tilde{r}^{2}}-\tilde{m}\right]^{2}}, \\
& \mathcal{F}_{I I}=\frac{-4 k_{1} \tilde{m}^{2} a^{2}}{k^{2}\left[(|\tilde{z}|+1)^{2}+\tilde{r}^{2}\right]\left[\sqrt{(|\tilde{z}|+1)^{2}+\tilde{r}^{2}}-\tilde{m}\right]^{2}} .
\end{aligned}
$$


We see that there exists a singularity at the surface determined by the equation

$$
(|\tilde{z}|+1)^{2}+\tilde{r}^{2}=\tilde{m}^{2}
$$

where all the non-trivial invariants diverge. The singularity equation allows real solutions only for $\tilde{m}>1$. On the other hand, in the previous subsection we showed that the energy condition for the disk-halo configuration implies that $\tilde{m}<1$. In fact, we can see from the expressions for the matter and charge density of the halo that they both diverge at the singular surface. Such divergencies are usually associated with the collapse of the gravitational configuration. This is an interesting result because it shows that the spacetime becomes singular as soon as the energy condition is violated. From a physical point of view this means that a disk-halo system can be constructed only if the condition $m / a<1$ is satisfied. As noticed in the last subsection, this condition implies an upper bound on the value of the mass parameter $m$ which is determined by the position of the mass along the symmetry axis. This, again, might be interpreted as a Chandrasekhar-like limit for the disk-halo system.

\section{CONCLUDING REMARKS}

In this work, we derived a relativistic model describing a thin disk surrounded by a halo in presence of an electromagnetic field. The model was obtained by solving the Einstein-Maxwell equations on a particular conformastatic spacetime in which only one independent metric function appears. For the energy-momentum tensor we used the distributional approach, and represented it as the sum of two distributional contributions, one due to the electromagnetic part and the other associated with a matter distribution. These assumptions allowed us to derive explicit expressions for the energy, pressure, electric current and electromagnetic field of the disk region and the halo as well. The main point of this approach is that it allows to write the gravitational and electromagnetic potentials in terms of a solution of Laplace's equation.

As a particular example, we used one of the simplest solutions of Laplace's equation, known as the Kuzmin solution, which contains two independent parameters, namely, the mass $m$ and the parameter $a$ that determines the proper length of the mass system. The resulting Kuzmin-like solution contains four independent parameters which determine the mass, proper length of the seed Kuzmin solution, electric charge and the magnetic field. The solution is asymptotically flat in general and turns out to be free of singularities if the ratio $m / a<1$, an inequality that also guarantees the fulfillment of all the energy conditions. We interpret this condition as a Chandrasekhar-like limit for the disk-halo system.

Since all the relevant quantities show a physically reasonable behavior within the range $m / a<1$, we conclude that the solution presented here can be used to describe the gravitational and electromagnetic fields of a think disk surrounded by a halo in the presence of an electromagnetic field.

Furthermore, with the particular solution of Laplace's equation here choosen, the matter distribution both of the disk and the halo vanishes sufficiently fast as $r$ and $z$ increase. This can be considered as indicating that the sources, the halo and the disk, are of finite size. Now, this reasonable behavior is a consequence of the behavior of the Kuzmin solution, which describes a gravitational potential that have a negative maximum at the center of the source and then decreases in absolute value, vanishing at infinity. Accordingly, we can expect that in a more general case a similar behavior will be obtained if solutions of the Laplace equation are considered that correspond to concentrated Newtonian sources with decreasing gravitational potential and gravitational field.

\section{ACKNOWLEDGMENTS}

One of us (A.C.G-P.) wants to thank COLCIENCIAS, Colombia, and TWAS-Conacyt for support. GAG is supported by DIEF de Ciencias (UIS), Grant No. 5189. HQ thanks DGAPA-UNAM for support. This work was supported in part by DGAPA-UNAM, Grant No. 106110, and Conacyt, Grant No. 166391.

\section{Appendix A: Appendix}

For the general comformastatic metric

$$
\mathrm{d} s^{2}=-\mathrm{e}^{2 \phi} \mathrm{d} t^{2}+\mathrm{e}^{2 \psi}\left[r^{2} \mathrm{~d} \varphi^{2}+\mathrm{d} r^{2}+\mathrm{d} z^{2}\right],
$$


the nonzero components of the Einstein tensor read

$$
\begin{aligned}
G_{00}^{ \pm} & =-e^{2(\phi-\psi)}\left\{2\left(\psi_{, r r}+\psi_{, z z}+\frac{1}{r} \psi_{, r}\right)+\psi_{, r}^{2}+\psi_{, z}^{2}\right\} \\
G_{11}^{ \pm} & =r^{2}\left\{\phi_{, r r}+\phi_{, r}^{2}+\phi_{, z z}+\phi_{, z}^{2}+\psi_{, r r}+\psi_{, z z}\right\} \\
G_{22}^{ \pm} & =\phi_{, z z}+\frac{1}{r} \phi_{, r}+\phi_{, z}^{2}+\psi_{, z z}+\frac{1}{r} \psi_{, r}+\psi_{, r}^{2}+2 \phi_{, r} \psi_{, r} \\
G_{23}^{ \pm} & =-\phi_{, r z}-\phi_{, r} \phi_{, z}+\phi_{, r} \psi_{, z}+\phi_{, z} \psi_{, r}-\psi_{, r z}+\psi_{, r} \psi_{, z} \\
G_{33}^{ \pm} & =\phi_{, r r}+\frac{1}{r} \phi_{, r}+\phi_{, r}^{2}+\psi_{, r r}+\frac{1}{r} \psi_{, r}+\psi_{, z}^{2}+2 \phi_{, z} \psi_{, z} .
\end{aligned}
$$

Furthermore, the nonzero components of the electromagnetic tensor (10) are given by

$$
\begin{aligned}
& E_{00}^{ \pm}=\frac{1}{2} e^{-2 \psi}\left\{A_{0, r}^{2}+A_{0, z}^{2}+r^{-2} e^{2(\phi-\psi)}\left(A_{, r}^{2}+A_{, z}^{2},\right)\right\} \\
& E_{01}^{ \pm}=e^{-2 \psi}\left(A_{0, r} A_{, r}+A_{0, z} A_{, z}\right) \\
& E_{11}^{ \pm}=\frac{1}{2} e^{-2 \psi}\left\{A_{, r}^{2}+A_{, z}^{2}+r^{2} e^{-2(\phi-\psi)}\left(A_{0, r}^{2}+A_{0, z}^{2}\right)\right\} \\
& E_{22}^{ \pm}=\frac{1}{2}\left\{-e^{-2 \phi}\left(A_{0, r}^{2}-A_{0, z}^{2}\right)+r^{-2} e^{-2 \psi}\left(A_{, r}^{2}-A_{, z}^{2}\right)\right\} \\
& E_{23}^{ \pm}=-e^{-2 \phi} A_{0, r} A_{0, z}+r^{-2} e^{-2 \psi} A_{, r} A_{, z}, \\
& E_{33}^{ \pm}=\frac{1}{2}\left\{e^{-2 \phi}\left(A_{0, r}^{2}-A_{0, z}^{2}\right)-r^{-2} e^{-2 \psi}\left(A_{, r}^{2}-A_{, z}^{2}\right)\right\}
\end{aligned}
$$

where, all the quantities are evaluated in the $z \geq 0$ and $z \leq 0$ regions. Consequently, from the general equation

$$
G_{a b}^{ \pm}=R_{a b}^{ \pm}-\frac{1}{2} g_{a b} R^{ \pm}=E_{a b}^{ \pm}+M_{a b}^{ \pm}
$$

and Eqs. (A2) and (A3), we have for the nonzero components of the matter energy-momentum tensor

$$
\begin{aligned}
& M_{00}^{ \pm}=-e^{2(\phi-\psi)}\left\{2 \nabla^{2} \psi+\nabla \psi \cdot \nabla \psi+\frac{1}{2} e^{-2 \phi} \nabla A_{0} \cdot \nabla A_{0}+\frac{1}{2} r^{-2} e^{-2 \psi} \nabla A \cdot \nabla A\right\}, \\
& M_{01}^{ \pm}=-e^{-2 \psi} \nabla A_{0} \cdot \nabla A, \\
& M_{11}^{ \pm}=r^{2}\left\{\nabla^{2}(\phi+\psi)-\frac{1}{r}(\phi+\psi)_{, r}+\nabla \phi \cdot \nabla \phi-\frac{1}{2} e^{-2 \phi} \nabla A_{0} \cdot \nabla A_{0}-\frac{1}{2} r^{-2} e^{-2 \psi} \nabla A \cdot \nabla A\right\} \\
& M_{22}^{ \pm}=\nabla^{2}(\phi+\psi)-(\phi+\psi)_{, r r}+\phi_{, z}^{2}+\psi_{r}^{2}+2 \phi_{, r} \psi_{r}+\frac{1}{2} e^{-2 \phi}\left(A_{0, r}^{2}-A_{0, z}^{2}\right)-\frac{1}{2} r^{-2} e^{-2 \psi}\left(A_{, r}^{2}-A_{, z}^{2}\right), \\
& M_{23}^{ \pm}=-(\phi+\psi)_{r z}-\phi \phi_{r}(\phi-\psi)_{, z}+\psi_{, r}(\phi+\psi)_{, z}+e^{-2 \phi} A_{0, r} A_{0, z}-r^{-2} e^{-2 \psi} A_{, r} A_{, z}, \\
& M_{33}^{ \pm}=\nabla^{2}(\phi+\psi)-(\phi+\psi)_{, z z}+\phi_{, r}^{2}+\psi_{, z}^{2}+2 \phi_{, z} \psi, z-\frac{1}{2} e^{-2 \phi}\left(A_{0, r}^{2}-A_{0, z}^{2}\right)+\frac{1}{2} r^{-2} e^{-2 \psi}\left(A_{, r}^{2}-A_{, z}^{2}\right) .
\end{aligned}
$$

Alternatively, the components of the MEMT in the local orthonormal tetrad for the general metric A1 can be 
expressed as

$$
\begin{aligned}
M_{(0)(0)}^{ \pm} & =-e^{-2 \psi}\left\{2\left(\psi_{, r r}+\psi_{, z z}+\frac{1}{r} \psi_{, r}\right)+\psi_{, r}^{2}+\psi_{, z}^{2}+\frac{1}{2} e^{-2 \phi}\left(A_{0, r}^{2}+A_{0, z}^{2}\right)+\frac{1}{2} r^{-2} e^{-2 \psi}\left(A_{, r}^{2}+A_{, z}^{2},\right)\right\} \\
M_{(0)(1)}^{ \pm} & =-r^{-1} e^{-(\phi+3 \psi)}\left(A_{0, r} A_{, r}+A_{0, z} A_{, z}\right), \\
M_{(1)(1)}^{ \pm} & =e^{-2 \psi}\left\{\phi_{, r r}+\phi_{, r}^{2}+\phi_{, z z}+\phi_{, z}^{2}+\psi_{, r r}+\psi_{, z z}-\frac{1}{2} e^{-2 \phi}\left(A_{0, r}^{2}+A_{0, z}^{2}\right)-\frac{1}{2} r^{-2} e^{-2 \psi}\left(A_{, r}^{2}+A_{, z}^{2}\right)\right\} \\
M_{(2)(2)}^{ \pm} & =e^{-2 \psi}\left\{\phi_{, z z}+\frac{1}{r} \phi_{, r}+\phi_{, z}^{2}+\psi_{, z z}+\frac{1}{r} \psi_{, r}+\psi_{, r}^{2}+2 \phi_{, r} \psi_{, r}+\frac{1}{2} e^{-2 \phi}\left(A_{0, r}^{2}-A_{0, z}^{2}\right)\right. \\
& \left.-\frac{1}{2} r^{-2} e^{-2 \psi}\left(A_{, r}^{2}-A_{, z}^{2}\right)\right\} \\
M_{(2)(3)}^{ \pm} & =e^{-2 \psi}\left\{-\phi_{, r z}-\phi_{, r} \phi_{, z}+\phi_{, r} \psi_{, z}+\phi_{, z} \psi_{, r}-\psi_{, r z}+\psi_{, r} \psi_{, z}+e^{-2 \phi} A_{0, r} A_{0, z}-r^{-2} e^{-2 \psi} A_{, r} A_{, z}\right\} \\
M_{(3)(3)}^{ \pm} & =e^{-2 \psi}\left\{\phi_{, r r}+\frac{1}{r} \phi_{, r}+\phi_{, r}^{2}+\psi_{, r r}+\frac{1}{r} \psi_{, r}+\psi_{, z}^{2}+2 \phi_{, z} \psi_{, z}-\frac{1}{2} e^{-2 \phi}\left(A_{0, r}^{2}-A_{0, z}^{2}\right)\right. \\
& \left.+\frac{1}{2} r^{-2} e^{-2 \psi}\left(A_{, r}^{2}-A_{, z}^{2}\right)\right\}, \\
\hat{J}_{(0)}^{ \pm} & =r e^{\psi}\left\{\nabla^{2} A_{0}-\nabla A_{0} \cdot \nabla(\phi-\psi)\right\}, \\
\hat{J}_{(1)}^{ \pm} & =-e^{\phi}\left\{\nabla^{2} A+\nabla A_{i} \nabla(\phi-\psi)-\frac{2 A_{, r}}{r}\right\} .
\end{aligned}
$$

[1] W.B. Bonnor, A. Sackfield, Communications in Mathematical Physics 8, 338 (1968)

[2] T. Morgan, L. Morgan, Physical Review 183, 1097 (1969)

[3] L. Morgan, T. Morgan, Physical Review D 2, 2756 (1970)

[4] B.H. Voorhees, Physical Review D 5, 2413 (1972)

[5] D. Lynden-Bell, S. Pineault, Mon. Not. R. Astron. Soc. 185, 679 (1978)

[6] A. Chamorro, R. Gregory, J.M. Stewart, Royal Society of London Proceedings Series A 413, 251 (1987)

[7] P.S. Letelier, S.R. Oliveira, Journal of Mathematical Physics 28, 165 (1987)

[8] J.P.S. Lemos, Classical and Quantum Gravity 6, 1219 (1989)

[9] J. Bičák, D. Lynden-Bell, J. Katz, Physical Review D 47, 4334 (1993)

[10] J. Bičák, D. Lynden-Bell, C. Pichon, Mon. Not. R. Astron. Soc. 265, 126, (1993)

[11] G.A. González, P.S. Letelier, Classical and Quantum Gravity 16, 479 (1999)

[12] G.A. González, O.A. Espitia, Physical Review D 68(10), 104028 (2003)

[13] G.A. González, A.C. Gutiérrez-Piñeres, V.M. Viña-Cervantes, Physical Review D 79(12), 124048 (2009)

[14] D. Lynden-Bell, S. Pineault, Mon. Not. R. Astron. Soc. 185, 695 (1978)

[15] J. Bičák, T. Ledvinka, Physical Review Letters 71, 1669 (1993)

[16] C. Pichon, D. Lynden-Bell, Mon. Not. R. Astron. Soc. 280, 1007 (1996)

[17] G.A. González, P.S. Letelier, Physical Review D 62(6), 064025 (2000)

[18] G. A.González and A. C. Gutiérrez-Piñeres, Classical and Quantum Gravity, 29(13),135001 (2012)

[19] J.P.S. Lemos, P.S. Letelier, Classical and Quantum Gravity 10, L75 (1993)

[20] J.P.S. Lemos, P.S. Letelier, Physical Review D 49, 5135 (1994)

[21] J.P.S. Lemos, P.S. Letelier, International Journal of Modern Physics D 5, 53 (1996)

[22] O. Semerák, M. Žáček, Classical and Quantum Gravity 17, 1613 (2000)

[23] O. Semerák, Classical and Quantum Gravity 19, 3829 (2002).

[24] M. Žáček, O. Semerák, Czechoslovak Journal of Physics 52, 19 (2002)

[25] O. Semerák, Classical and Quantum Gravity 20, 1613 (2003)

[26] O. Semerák, Classical and Quantum Gravity 21, 2203 (2004)

[27] V. Karas, J. Huré, O. Semerák, Classical and Quantum Gravity 21, 1 (2004)

[28] A. C.Gutiérrez-Piñeres, G.García-Reyes and G. A. González, ArXiv e-prints, 1209.0500 (2012)

[29] A. Feinstein, J. Ibañez, R. Lazkoz, Ap. J. 495, 131 (1998)

[30] D. Vogt, P.S. Letelier, Physical Review D 68(8), 084010 (2003)

[31] M. Ujevic, P.S. Letelier, Physical Review D 70(8), 084015 (2004)

[32] T. Ledvinka, M. Žofka, J. Bičák, in Recent Developments in Theoretical and Experimental General Relativity, Gravitation, and Relativistic Field Theories, T. Piran \& R. Ruffini (1999)

[33] G. García-Reyes, G.A. González, Brazilian Journal of Physics 37, 1094 (2007)

[34] P.S. Letelier, Physical Review D 60(10), 104042 (1999).

[35] A. C. Gutiérrez-Piñeres and G. A. González, International Journal of Theoretical Physics, 51,1737-1752 (2012) 
[36] D. Vogt, P.S. Letelier, Classical and Quantum Gravity 21, 3369 (2004)

[37] J. Katz, J. Bičák, D. Lynden-Bell, Classical and Quantum Gravity 16, 4023 (1999)

[38] G.A. González, A.C. Gutiérrez-Piñeres, P.A. Ospina, Physical Review D 78(6), 064058 (2008)

[39] G. García R., G.A. González, Physical Review D 69(12), 124002 (2004)

[40] D. Vogt, P.S. Letelier, Physical Review D 70(6), 064003 (2004)

[41] G. García-Reyes, G.A. González, Classical and Quantum Gravity 21, 4845 (2004)

[42] G. García-Reyes, G.A. González, Physical Review D 70(10), 104005 (2004)

[43] N. Gürlebeck, J. Bičák and A. C. Gutiérrez-Piñeres, Physical Review D 83(12), 124023 (2011)

[44] N. Gürlebeck, J. Bičák and A. C. Gutiérrez-Piñeres, General Relativity and Gravitation, 43,3301-3312(2011)

[45] J. L. Synge, Relativity: The General Theory. (North-Holland, Amsterdam, 1966).

[46] H. Stephani, D. Kramer, M. McCallum, C. Hoenselaers, and E. Herlt Exact Solutions of Einsteins's Field Equations (Cambridge University Press, Cambridge, England, 2003).

[47] G. A. González and R. Vera, Classical and Quantum Gravity 28, 025008 (2011).

[48] A. Papapetrou and A. Hamouni, Ann. Inst. Henri Poincaré 9, 179 (1968).

[49] A. Lichnerowicz, C.R. Acad. Sci. 273, 528 (1971).

[50] A. H. Taub, J. Math. Phys. 21, 1423 (1980).

[51] E. Israel, Nuovo Cimento 44B, 1 (1966)

[52] E. Israel, Nuovo Cimento 48B, 463 (1967)

[53] E. Poisson, A Relativist's Toolkit: The Mathematics of Black-Hole Mechanics. (Cambridge University Press, 2004)

[54] G. G. Kuzmin, Astron. Zh., 33, 27 (1956)

[55] J. Binney and S. Tremaine, Galactic Dynamics. (Princeton University Press, 1987)

[56] C. Cherubini, D. Bini, S. Capozziello and R. Ruffini, Int. J. Mod. Phys. D 11, 827 (2002) 